\title{
Addressing the causes and consequences of disruptive behaviors in the healthcare setting
}

\begin{abstract}
Disruptive behaviors in health care can have a significant adverse effect on staff interactions that can negatively impact staff satisfaction, staff performance, and patient outcomes of care. Despite growing evidence of the ill effects of these types of behaviors many organizations are still having a difficult time in addressing these issues in an effective manner. Gaining a better understanding of the nature, causes, and impact of these behaviors is crucial to finding the right remedies for solution. Nobody intentionally starts the day planning to be disruptive; it's just that things get in the way. A combination of deep seated factors related to age and gender preferences, culture and ethnicity, life experiences, and other events that help shape values, attitudes and personalities, and more external factors related to training, environmental pressures, and personal issues all contribute to the mix. Early recognition, early intervention, and taking a pro-active supportive approach to improve individual behaviors will result in better relationships, less disruption, more satisfaction, and better outcomes of care.
\end{abstract}

Keywords: disruptive behaviors, staff relationships, patient outcomes
Volume 3 Issue 3 - 2015

Alan H Rosenstein

Health care consultant, USA

Correspondence: Alan H Rosenstein, 139 I5th Avenue, San Francisco, CA 94II8, USA, Tel 4I53707754,

Email ahrosensteinmd@aol.com

Received: June 19,2015 | Published: August 17,2015

\section{Introduction}

Disruptive behaviors occur across all professions and all walks of life, but when they occur in the health care setting it takes on added significance as to the potential for patient harm. ${ }^{1}$ Despite increasing public notoriety of the ill effects of bullying and intimidation in the workplace, many health care organizations still have difficulty in dealing with this issue in an effective manner. ${ }^{2-4}$ Barriers may include unawareness of the seriousness of the issue, tolerance, reluctance to intervene, or an inefficient structure, process, or skill set needed to take appropriate action. Gaining a better understanding of the causes and consequences of disruptive events is crucial in order to put together a program designed to raise awareness, establish accountability, and provide resources to reduce its occurrence.

\section{Precipitating factors}

In the health care setting disruptive behaviors are defined as any inappropriate behavior that can negatively impact patient care and undermine a culture of patient safety. ${ }^{5,6}$ Most of the behaviors reported describe such behaviors as abusive language, condescending or berating remarks, harassment, or other forms of intimidation. Actual physical insult is a rare occurrence. Gaining a better understanding of underlying factors contributing to individual values, perceptions, and attitudes that may trigger a disruptive event is a crucial component in looking for opportunities to lessen the likelihood of occurrence (Table1).

On one side are the more entrenched deep seated factors influencing behavioral tendencies. Age and generational values, gender based tendencies, the influence of culture and diversity on views about gender, hierarchy, and communication styles, and the accumulation of other life experiences all contribute to perceptions, values, subconscious biases, and attitudes that affect ones personality. On the other side is the influence of more adult related experiences. These include the training environment, and influences of any current environmental, work related, and/or personal life experiences that shape one's mood and disposition. All of the factors need to be considered when looking for ways to modify unprofessional behaviors.

\section{Resolution}

Table 1 provides a structure for getting things done. The first step is to recognize if there is an issue. You can start by reviewing the number and the nature of registered complaints, or better yet, distribute a confidential staff survey to assess staff views on the status of the work environment. The organization needs to be willing and able to respond to any appropriate complaints or issues that are raised through their internal assessment. This requires a strong culture and leadership commitment to holding all personnel to abide by a professional code of conduct with a zero tolerance policy toward behaviors that could potentially negatively impact staff performance or patient care.

While the intent may be here, there are several potential obstacles that may inhibit willingness to proceed. First is traditional organizational hierarchy where physicians are regarded and treated as a separate autonomous entity ("sacred saints") and organizations are afraid of crossing boundaries. Second is the fear of antagonizing a physician who may threaten to bring his or her patients (and revenues) elsewhere. Next is an overriding "code of silence" where physicians and others are reluctant to report inappropriate behaviors. And finally is the lack of structure, reporting process, and skill sets needed to conduct a thorough investigation, review, and initiate follow actions. These are all organizational culture issues that need to be addressed before moving forward.

The best remedy is prevention. Looking back to the internal factors affecting physician behavior (Table 1) there are a number of different options available to provide appropriate education and training programs in an effort to improve relationship dynamics. Offering programs on diversity management, cultural competency, generational differences, harassment, conflict management, and "personalysis" can help individuals better recognize behavioral influences and values in both themselves and others. In a previous issue of this journal we emphasized the importance of teaching emotional intelligence as a way to improve understanding and reactions affecting interpersonal dynamics. ${ }^{7}$ After teaching the why and how best to react curriculum the organization then needs to follow up by providing training 
programs designed to improve communication and team collaboration skills. This is particularly important for the physician population. ${ }^{8}$

Focusing on the external factors, there may be an even greater opportunity for improvement as many of the issue are less deep seated.

Table I Factors Affecting Behaviors

\begin{tabular}{ll}
\hline Internal & External \\
\hline Age (generation) & Training \\
\hline Gender & Environmental Factors \\
\hline Culture \& Ethnicity & Work \\
\hline Geographics/ Life Experiences & Social/ Personal \\
\hline Personality Profile & Reality \& Expectations \\
\hline
\end{tabular}

Table 2 Solutions

\begin{tabular}{ll}
\hline I & Organizational Awareness \\
2 & Organizational Commitment \\
3 & Address Barriers \\
4 & Education and Training \\
5 & Physician Engagement \\
6 & Policies and Procedures \\
7 & Intervention \\
\hline
\end{tabular}

Many of the problems start in the medical training environment Some equate this to an atmosphere of "hazing" similar to experiences in pledging college fraternities. You start out with low self- esteem, develop competency through exhaustive independent study, and wind up delivering care in an autonomous dictatorial manner. Physicians give orders. The focus is on gaining knowledge and procedural competency rather than developing people and relationship skills. Fortunately this is beginning to change. There is now a dedicated movement to attract more "well- rounded" students who have focused on the social sciences and humanities and not just math and science. The new MCAT tests (Medical College Admission Test) has added a new series of questions that focus on social science. ${ }^{9}$ Many of the more progressive medical schools are beginning to change their core curriculum to emphasize the importance of team collaboration and interdisciplinary training. ${ }^{10,11}$

One of the strongest contributors to inappropriate behaviors is the growing impact of stress and burnout in health care professions. With concerns about increasing complexity, the introduction of new technologies, health care reform, changing modes of delivery and models of care, greater external scrutiny and accountability for performance outcomes, and a growing perception of loss of autonomy and control, more than $50 \%$ of physicians report significant levels of stress and burnout that are negatively affecting their anxieties and attitudes toward medical practice. ${ }^{12}$ Organizations need to recognize the impact of stress and burnout on performance and provide appropriate support. Providing courses on stress management or wellness is a start but offering more customized services in the way of individualized coaching and counseling provided by either Human Resources, Physician Wellness Committees, Physician EAPs (Employee Assistance Programs), or though other dedicated resources have proved of particular benefit in helping physicians better adjust to the stress and pressures of providing care. These efforts frequently result in improved work relationships, increased satisfaction, a lower likelihood of a disruptive event, and helps restore their passion for care. ${ }^{13}$ Another corollary approach is to expand efforts to increase overall physician engagement by setting up a consistent process of listening and responding to their concerns and providing appropriate logistical, clinical, and behavioral support. Allowing physicians to have input, involving them in process redesign, and providing needed support increases their likelihood of behavioral compliance. ${ }^{14}$

To promote professional behaviors the organization needs to have in place a set of (Table 2) policies and procedures defining a professional code of conduct and outline the process to be followed for those who don't comply. Physicians need to sign off on agreeing with this policy as part of their credentialing or employment agreement. If incidents occur there needs to be a consistent reporting policy where all complaints are reviewed in a non- biased confidential manner. Follow up interventions need to be conducted by individuals skilled in communication and facilitation techniques and be able to make appropriate recommendations for next steps.

There are several different levels of intervention. The first is just to raise awareness. Many individuals are not aware of their actions being construed as disruptive behaviors and are unaware of the potential for ill downstream effects. Raising awareness and making suggestions on how they could have better handled the situation will enable many of these individuals to self-correct. More troublesome complaints require a formal approach. Recommendations may include that they take courses on anger, stress, conflict, or diversity management, seek coaching or counseling services, or in some cases, be referred to programs that specialize in managing disruptive behaviors. Some individuals have deep seated behavioral disorders and will require psychiatric services. Always keep in mind the possibility of underlying substance abuse. In more extreme cases involving physicians who are resistant to change curtailing privileges, sanctions, or termination may be the only viable action.

\section{Recommendations}

Disruptive behaviors can lead to a toxic workplace environment that can have a profound effect on staff relationships and patient outcomes of care. Organizations need to be willing to address these issues in an appropriate effective manner. The best approach is to gain a better understanding of what motivates attitudes and behaviors and provide appropriate training modules designed to improve behavioral understanding and reaction to enhance staff and patient relationships in an effort to avoid a disruptive event. Stress and burnout are a significant contributing factor and providing services to help providers adjust to the pressures of medical practice will go a long way in improving their attitudes, outlooks, and behaviors. Most importantly we need to look at physicians and all medical personnel as a precious over worked over extended resource and do what we can to help them thrive. They are often too consumed to seek help on their own so we must take the initiative by providing any needed support and promoting an acceptable work-life balance to enhance their own well-being.

\section{Acknowledgments}

None.

\section{Conflicts of interest}

Author declares there are no conflicts of interest.

\section{Funding}

None.

\section{References}

1. Rosenstein A, O'Daniel M. A Survey of the Impact of Disruptive Behaviors and Communication Defects on Patient Saf. Jt Comm J Qual Patient Saf. 2008;34(8):464-471. 
2. Disrespectful Behavior in Healthcare: Have We Made any Progress in the Last Decade. ISMP Medication Safety Alert . 2013;11(7):1-4.

3. Sanchez L. Disruptive Behaviors among Physicians. JAMA. 2014;312(21):2209-2210.

4. Rosenstein AH. Bad Medicine: Managing the Risks of Disruptive Behaviors in Health Care Settings. RiskManagement. 2013;60(10):38-42.

5. AMA. Medical Staff Code of Conduct. 2012.

6. Leadership Standard Clarified to Address Behaviors That Undermine a Safety Culture. Joint Commission Perspective . 2012;32(1):7.

7. Rosenstein A, Stark D. Emotional Intelligence: A Critical Tool to Understand and Improve Behaviors That Impact Patient Care. Journal of Psychology and Clinical Psychiatry. 2015;2(1):1-4.

8. Rosenstein AH. Physician Communication and Care Management: The Good, the Bad, and the Ugly. Physician Exec. 2012;38(4):34-37.

9. Beck M. Innovation is sweeping through U.S. Medical Schools. The Wall Street Journal. 2014.
10. Brock D, Abu-Rish E, Chiu CR, et al. Interprofessional Education in Team Communication: Working Together to Improve Patient Safety. Postgrad Med J . 2013;89(1057):642-651.

11. Muller D. Reforming Pre-Medical Education: Out With the Old, In With the New. New England Journal of Medicine. 2013;368(17):1567-1569.

12. Shanafelt TD, Boone S, Tan L, et al. Burnout and Satisfaction With Work-life Balance Among US Physicians Relative to the General US Population. Arch Intern Med . 2012;172(18):1377-1385.

13. Danielson D, Ketterling R, Rosenstein A. Physician Stress and Burnout: Causes, Effects, and Impact on Performance and Behavior. AMGA Group Practice Journal. 2013;62(3):38-41.

14. Rosenstein A.Meeting the Physician's Needs: The Road to Organizational-Physician Engagement. Trustee. 2015;19-22. 